\title{
IGNITION OF A REACTIVE SOLID EXPOSED TO A STEP IN SURFACE TEMPERATURE*
}

\author{
A. LIÑÁN† AND F. A. WILLIAMS
}

\begin{abstract}
The title problem is classical in ignition theory. Here it is solved by an asymptotic analysis in which the nondimensional activation energy is treated as a large parameter. Inert and reactive zones are identified, and a temperature peak develops in the latter during a stage of transition to ignition. A criterion of thermal runaway yields a formally correct asymptotic expansion for the ignition time which agrees with results of numerical integrations if the activation energy is sufficiently large.
\end{abstract}

1. Introduction. The problem of ignition of a semi-infinite reactive solid whose surface temperature is increased at time zero and thereafter held constant has been reviewed by Merzhanov and Averson [1]. The mathematical statement of the problem is

$$
\begin{array}{cc}
\rho c \frac{\partial T}{\partial t}=\lambda \frac{\partial^{2} T}{\partial x^{2}}+\rho q \nu e^{-E / R T} & (x>0, \quad t>0), \\
T(x, 0)=T \infty, t)=T_{i}, & T(0, t)=T_{s},
\end{array}
$$

where $T_{s}$ and $T_{i}$ are positive constants, with $T_{s}>T_{i}$. The density $\rho$, specific heat $c$, thermal conductivity $\lambda$, heat of reaction $q$, pre-exponential rate factor $\nu$, activation energy $E$ (and universal gas constant $R$ ) all are assumed to be positive constants. Numerical integrations have been performed to obtain profiles in the space coordinate $x$, of temperature $T$, as functions of time $t$. Approximate analytical correlations for the time to ignition have been developed from the results [1].

Recently we have been applying asymptotic analysis, treating a nondimensionalized $E$ as a large parameter, to obtain analytical solutions to problems like that defined by (1) and (2) [2], [3]. In particular, we have solved the corresponding problem for the case of a constant energy flux applied to the surface [2]. The case of constant surface temperature may be viewed as a special one for which the analysis of [2], generalizable to a wide variety of flux-time histories [3], cannot be applied. The solution to this comparatively difficult problem is developed herein.

It is useful to nondimensionalize the problem. Two independent nondimensional parameters appear, viz., $\alpha=\left(T_{s}-T_{i}\right) / T_{s}$ and $\varepsilon=R T_{s}^{2} /\left[E\left(T_{s}-T_{i}\right)\right]$. We choose to define nondimensional time, space and temperature variables as $\tau=$ $t\left(2 \pi \varepsilon q \nu /\left(D c T_{s} \alpha\right)\right) e^{-E / R T_{s}}, \quad \xi=x\left(2 \pi \varepsilon \rho q \nu /\left(D \lambda T_{s} \alpha\right)\right)^{1 / 2} e^{-E / 2 R T_{s}} \quad$ and $\theta=\left(T-T_{s}\right) \cdot$ $\left(E /\left(R T_{s}^{2}\right)\right)$ where $D$ is a nondimensional parameter whose value will be selected so that ignition occurs at $\tau=1$. Equations (1) and (2) become

$$
\frac{\partial \theta}{\partial \tau}-\frac{\partial^{2} \theta}{\partial \xi^{2}}=D(2 \pi)^{-1} \varepsilon^{-2} e^{\theta /(1+\alpha \varepsilon \theta)} \quad(\xi>0, \tau>0)
$$

* Received by the editors January 28,1978 . This work was supported partially by the United States Army Research Office, Durham under Grant AROD-31-124-73-G108. Later portions of the work were supported by the United States Air Force Office of Scientific Research under Grant AFOSR 77-3362.

$\dagger$ Escuela de Ingenieros Aeronáuticos, Ciudad Universitaria, Madrid, Spain.

\$ Department of Applied Mechanics and Engineering Sciences, University of California, San Diego, La Jolla, California 92093. 
and

$$
\theta(\xi, 0)=\theta(\infty, \tau)=-\varepsilon^{-1}, \quad \theta(0, \tau)=0 .
$$

Our objective is to develop an asymptotic expansion of the solution to (3) and (4), for small values of $\varepsilon$, with $\alpha$ of order unity. Uniformity in $\xi$ is required, but in order to define an ignition event in a most natural way [2], uniformity in $\tau$ is sought only prior to a time of thermal runaway, $\tau=1$. It will be assumed, as later verified from its asymptotic expansion ( $\$ 5)$, that $D$ is of order unity. An alternative approach, yielding the same results, would be to put $D=1$ and solve for the value of $\tau$ at which thermal runaway occurs. The present formulation facilitates carrying the analysis to higher orders.

2. Inert zone. From (3) and (4) it is clear that the reaction term becomes exponentially small as $\theta$ approaches $\theta(\infty, \tau)$. This suggests that there will exist an outer zone of inert heat conduction. In studying this zone, it is useful to work with the difference between the true solution and the solution to the inert problem whose boundary conditions are given by (4). This inert solution is $\theta_{I}=-\varepsilon^{-1} \operatorname{erf}(\zeta)$, where $\zeta=\xi /(2 \sqrt{\tau})$. It is also useful, although not essential, to work with $\zeta$ in place of $\xi$ as the spatial variable. With $\varphi(\zeta, \tau) \equiv \theta(\xi, \tau)-\theta_{I}(\zeta)$, equations (3) and (4) become

$$
4 \tau \frac{\partial \varphi}{\partial \tau}-2 \zeta \frac{\partial \varphi}{\partial \zeta}-\frac{\partial^{2} \varphi}{\partial \zeta^{2}}=\frac{2 D \tau}{\pi \varepsilon^{2}} \exp \left\{\frac{\varphi-\varepsilon^{-1} \operatorname{erf}(\zeta)}{1+\alpha \varepsilon\left[\varphi-\varepsilon^{-1} \operatorname{erf}(\zeta)\right]}\right\} \quad(\zeta>0, \quad \tau>0)
$$

and

$$
\varphi(\zeta, 0)=\varphi(\infty, \tau)=\varphi(0, \tau)=0
$$

which constitutes an alternative exact representation of the original problem. In the following, $\theta_{\mathrm{I}}(\zeta)+\varphi(\zeta, \tau)$ will denote the solution in the outer, inert zone.

The problem in the outer zone is defined by the heat equation in transformed variables, viz. (5) with the right hand side set equal to zero. Since the reaction term is exponentially small in the outer zone [2], terms arising therefrom will not be written in the expansion. In (6), the condition $\varphi(0, \tau)=0$ must be deleted and replaced by suitable conditions of matching to an inner zone. To avoid carrying along the differential equation for the outer zone, it is helpful to recall [4, p. 76], that there exists an integral relationship between the surface temperature and the surface temperature gradient, for the (homogeneous) transient heat equation in a semi-infinite slab. In terms of the variables $\tau$ and $\zeta$, the Abel inversion of this relationship is

$$
\varphi_{\zeta}(0, \tau)=-2 \sqrt{\frac{\tau}{\pi}} \int_{0}^{\tau} \frac{d \varphi\left(0, \tau^{\prime}\right)}{d \tau^{\prime}} \frac{d \tau^{\prime}}{\sqrt{\tau-\tau^{\prime}}}
$$

Equation (7) contains all of the information needed concerning the outer, nonreactive zone. It is used for matching of the solution obtained in the inner, reactive zone. In ( 7$), \varphi$ denotes the outer dependent variable, and the indicated position $\zeta=0$ signifies the matching region, not the true surface of the solid, so that $\varphi(0, \tau)$ and $\varphi_{\zeta}(0, \tau) /(2 \sqrt{\tau})$ represent the apparent increments above inert values for surface temperature and for surface heat-flux, respectively, as seen from the nonreactive zone.

3. Reaction zone. To analyze the inner region in which the reaction occurs, we introduce the stretched spatial variable $\eta=2 \sqrt{\tau / \pi} \zeta / \varepsilon$. With $\eta$ of order unity, the expansion of the error function,

$$
\operatorname{erf}(\zeta)=\frac{2}{\sqrt{\pi}} \sum_{n=0}^{\infty} \frac{(-1)^{n} \zeta^{2 n+1}}{n !(2 n+1)}
$$


may be used to derive from (5) the inner equation

$$
\frac{\partial^{2} \psi}{\partial \eta^{2}}=-\frac{D}{2} e^{\psi-\eta / \sqrt{ } \tau}\left[1-\alpha \varepsilon(\psi-\eta / \sqrt{\tau})^{2}\right],
$$

valid up to terms of order $\varepsilon^{2}$ when the appropriate number of terms in the asymptotic expansion of $D$ is retained. Here $\psi(\eta, \tau) \equiv \varphi(\zeta, \tau)$, the former symbol being used for the inner expansion. The time derivative does not appear in (9) because the stretching shows it to be of order $\varepsilon^{2}$. Although results of using (9) will be invalid when the difference between $\tau$ and the ignition time is of order $\varepsilon^{2}$, in this paper expansions will be carried only to order $\varepsilon^{3 / 2}$, and therefore (9) is sufficient for the present analysis. The applicable boundary condition obtained from (6) for (9) is $\psi(0, \tau)=0$, and matching with (7) must be imposed for large $\eta$. The $\alpha \varepsilon$ term represents the first correction for the departure of Arrhenius kinetics from Frank-Kamenetskii kinetics. Note that the expansion of the reaction term in (9) has modified the problem to one in which $\psi$ goes to infinity at a finite value of $\tau$, as is characteristic of Frank-Kamenetskii kinetics; this provides a natural definition of the ignition time [2].

Integration of (9) is facilitated by treating $\psi-\eta / \sqrt{\tau}$ as the dependent variable. It is convenient to denote this quantity by $\theta(\eta, \tau)$ since it equals the nondimensional temperature where a one-term expansion of (8) is applicable. Henceforth $\theta$ will denote this inner dependent variable, which is not precisely the same as the $\theta$ that appears in (3). The left-hand side of (9) can then be written as $\frac{1}{2}(\partial / \partial \theta)\left[(\partial \theta / \partial \eta)^{2}\right]$, and therefore a first integral readily is found to be

$$
\frac{\partial \theta}{\partial \eta}=\mp\left\{F^{2}+D(1-2 \alpha \varepsilon)-D e^{\theta}\left[1-\alpha \varepsilon\left(\theta^{2}-2 \theta+2\right)\right]\right\}^{1 / 2}
$$

where $F(\tau)$ is an integration constant denoting the $\eta$ dervative of $\theta$ evaluated at $\eta=0$. The nondimensional measure $F$ of the surface heat flux will begin at $-\infty$ at $\tau=0$ and will increase monotonically with $\tau$.

In (10) the upper sign is to be used at early times since the temperature gradient is negative then. Eventually a time $\tau_{a}$ is reached at which the temperature gradient vanishes at $\eta=0$. For $\tau>\tau_{a}$, the temperature peaks in the interior of the solid at a position identified by the subscript $m$. Since $\partial \theta / \partial \eta>0$ for $\eta<\eta_{m}$ and $\partial \theta / \partial \eta<0$ for $\eta>\eta_{m}$, if $\tau>\tau_{a}$ then the upper sign in (10) is to be used for $\eta>\eta_{m}$ and the lower sign for $\eta<\eta_{m}$. Since the transition from positive to negative slope must be continuous, the vanishing of the right hand side of $(10)$ provides an expression for the nondimensional maximum temperature $\theta_{m}(\tau)$, viz.,

$$
\theta_{m}=\ln \left(\frac{D+F^{2}}{D}\right)+\alpha \varepsilon\left\{\left[\ln \left(\frac{D+F^{2}}{D}\right)\right]^{2}-2 \ln \left(\frac{D+F^{2}}{D}\right)+\frac{2 F^{2}}{D+F^{2}}\right\},
$$

for $\tau>\tau_{a}$, through terms of order $\varepsilon$.

A variety of characteristics of the reaction-zone solution can be seen most easily by neglecting the $\alpha \varepsilon$ term, since this greatly simplifies integration of (10). The general integral becomes

$$
\eta=\frac{ \pm 1}{\sqrt{D+F^{2}}} \ln \left[\frac{1+\sqrt{1-D e^{\theta} /\left(D+F^{2}\right)}}{1-\sqrt{1-D e^{\theta} /\left(D+F^{2}\right)}}\right]+\eta_{m},
$$

where $\eta_{m}(\tau)$ is a constant of integration. From (11) it is seen that if $\alpha \varepsilon$ is neglected, then the value of $D e^{\theta} /\left(D+F^{2}\right)$ is unity at $\theta=\theta_{m}$; equation (12) therefore shows the constant $\eta_{m}$ to coincide with the value of $\eta$ at the point of maximum temperature when 
$\tau>\tau_{a}$. Since at $\eta=0$ the upper sign holds for $\tau<\tau_{a}$ and the lower for $\tau>\tau_{a}$, the boundary condition that $\theta=0$ at $\eta=0$ requires

$$
\eta_{m}=\frac{1}{\sqrt{D+F^{2}}} \ln \left(\frac{\sqrt{D+F^{2}}+F}{\sqrt{D+F^{2}}-F}\right)
$$

which is zero at $F=0$, reaches a maximum value of $2 / F$ at $F=1.51 \sqrt{D}$ (the solution to the transcendental equation $\left.\ln \left[\left(\sqrt{D+F^{2}}+F\right) /\left(\sqrt{D+F^{2}}-F\right)\right]=2 \sqrt{D+F^{2}} / F\right)$, and returns to zero as $F$ approaches infinity. Inversion of (12) shows that

$$
\theta=\ln \left\{\frac{(4 / D)\left(D+F^{2}\right) e^{ \pm\left(\eta-\eta_{m}\right) \sqrt{D+F^{2}}}}{\left[1+e^{ \pm\left(\eta-\eta_{m}\right) \sqrt{D+F^{2}}}\right]^{2}}\right\}
$$

The asymptotic solution for $\theta$ at large values of $\eta$ is needed in connection with matching. An expansion of (10) shows directly that

$$
\frac{\partial \theta}{\partial \eta} \sim-\sqrt{D+F^{2}}+\alpha \varepsilon / \sqrt{D+F^{2}}+O\left(\varepsilon^{2}\right)+\text { e.s.t. }
$$

where e.s.t. stands for terms that are exponentially small in $\eta$ (specifically, no larger than order $\theta^{2} e^{\theta}$ ). Equation (15) implies that $\theta \sim$ const. $\eta+$ const., and an expression for the second constant is needed. This expression may be obtained directly from the expansion of (14) if terms of order $\alpha \varepsilon$ are neglected. Since retention of the $\alpha \varepsilon$ term makes the derivation considerably more involved algebraically, only the result will be quoted. It is found by integrating (10) that

(16) $\theta \sim-\eta \sqrt{D+F^{2}}+\frac{D \alpha \varepsilon \eta}{\sqrt{D+F^{2}}}+\ln \left[\frac{4}{D}\left(D+F^{2}\right)\left(\frac{\sqrt{D+F^{2}}+F}{\sqrt{D+F^{2}}-F}\right)\right]+\alpha \varepsilon G+O\left(\varepsilon^{2}\right)+$ e.s.t.

where $G$ is a complicated function of $F / \sqrt{D}$ whose value and derivative remain bounded for $F / \sqrt{D}$ finite.

4. Integral equation for matching. Matching requires that for the parametric limit in which $\varepsilon$ goes to zero, in an intermediate variable the relationship $\varphi(\zeta, \tau) \sim \psi(\eta, \tau)$ must hold. This condition may be written more explicitly as

$$
\varphi(0, \tau)+\zeta \varphi_{\zeta}(0, \tau)+\cdots \sim \eta / \sqrt{\tau}+\theta,
$$

in which $\theta$ is to be given by (16). From the definitions of $\zeta$ and $\eta$ it may then be shown that in the lower orders matching necessitates

$$
\varphi_{\zeta}(0, \tau) \sim \frac{2}{\sqrt{\pi} \varepsilon}\left[1-\sqrt{\tau\left(D+F^{2}\right)}+D \alpha \varepsilon \sqrt{\tau /\left(D+F^{2}\right)}\right]
$$

and

$$
\varphi(0, \tau) \sim \ln \left[\frac{4}{D}\left(D+F^{2}\right)\left(\frac{\sqrt{D+F^{2}}+F}{\sqrt{D+F^{2}}-F}\right)\right]+\alpha \varepsilon G .
$$

Use of (18) and (19) in (7) produces an integral equation for generating the asymptotic expansion of the unknown function $F(\tau)$, namely

(20) $1-\sqrt{\tau\left(D+F^{2}\right)}+D \alpha \varepsilon \sqrt{\tau /\left(D+F^{2}\right)} \sim-2 \varepsilon \sqrt{\tau} \int_{0}^{\tau} \frac{d F^{\prime}}{d \tau^{\prime}}\left[\frac{F^{\prime}+\sqrt{D+F^{\prime 2}}}{D+F^{\prime 2}}\right] \frac{d \tau^{\prime}}{\sqrt{\tau-\tau^{\prime}}}$,

up to terms of order $\varepsilon^{2}$. Here $F^{\prime}$ denotes $F\left(\tau^{\prime}\right)$. 
The first approach to pursue in seeking to solve (20) is to attempt to construct a regular expansion of $F(\tau)$ in powers of $\varepsilon$. When this is done, it is found that

$$
F=-\sqrt{\frac{1-D \tau}{\tau}}-D \alpha \varepsilon \sqrt{\frac{\tau}{1-D \tau}}+\varepsilon \frac{\ln (1-D \tau)}{\sqrt{\tau} \sqrt{1-D \tau}}
$$

through terms of order $\varepsilon$. The deficiencies of this solution are evident in that its terms become imaginary for $D \tau>1$ and exhibit stronger divergences in higher-order terms for small values of $(1-D \tau)$. Although the ordinary expansion implies that $D \tau=1$ constitutes a first approximation for $\tau_{a}$ and that very likely $D=1$ in lowest order, (21) cannot be used to describe the solution in the vicinity of the ignition time or to obtain a better approximation for its value. However, a stretching of the time variable about $\tau=1$ may produce solutions of greater accuracy in the vicinity of the ignition time.

Therefore the variable $y=(1-\tau) / \varepsilon$ is introduced, and an expansion of the form $D=1+\delta$ is tried, where $\delta=o(1)$ as $\varepsilon$ approaches zero. Times such that $1-\tau$ is of order unity correspond to an initial stage during which (21) provides the solution, while times such that $y$ is of order unity correspond to a transition stage during which the ignition event occurs. The value of $F$ is small during the transition stage; so that the flux appears on both sides of (20) in lowest order, the stretching $f=F / \sqrt{\varepsilon}$ is employed.

When these stretched variables are substituted into (20) and a formal expansion in $\varepsilon$ is tried directly, the equation

$$
y-f^{2}-\delta / \varepsilon+2 \alpha \sim 4 \int_{y}^{1 / \varepsilon} \frac{d f^{\prime}}{d y^{\prime}} \frac{d y^{\prime}}{\sqrt{y^{\prime}-y}} .
$$

is obtained in lowest order, where $f^{\prime}$ denotes $f\left(y^{\prime}\right)$. For large $y$ the expected solution, consistent with (21), is $f=-\sqrt{y}$, but substitution of this into the integral produces in lowest order $2 \ln (\varepsilon y / 4)$ on the right-hand side. This logarithmic divergence may be shown to imply, through matching to the initial stage, that $f$ behaves like $-\sqrt{y}+\ln y / \sqrt{y}$ for large $y$. It is important to find a way to account properly for the logarithmic divergence in working with the integral equation.

Among a number of possible procedures, one approach, correct in low orders, is first to employ the identity

$$
\int_{0}^{\tau} \frac{\left(1-\sqrt{1-\tau^{\prime}}\right)\left(\tau / \tau^{\prime}\right)}{\sqrt{\tau^{\prime}\left(1-\tau^{\prime}\right)} \sqrt{\tau-\tau^{\prime}}} d \tau^{\prime}=-\ln (1-\tau)
$$

for the purpose of subtracting the divergence. If (22) is multiplied by $\varepsilon$ and added to (20), then after the variables $y$ and $f$ are introduced an expansion in $\varepsilon$ formally produces

$$
\begin{aligned}
& y-f^{2}-2 \ln (\varepsilon y)-\delta / \varepsilon+2 \alpha+O(\delta, \varepsilon) \\
& \sim \int_{y}^{K}\left[\frac{2}{\sqrt{y^{\prime}}}+4 \frac{d f^{\prime}}{d y^{\prime}}-2 \sqrt{\varepsilon}\left(1-2 f^{\prime} \frac{d f^{\prime}}{d y^{\prime}}\right)\right] \frac{d y^{\prime}}{\sqrt{y^{\prime}-y}} .
\end{aligned}
$$

The upper limit $K$ of the integral, to be selected to effect matching with the initial stage, must be large compared with unity but small compared with $1 / \varepsilon$.

5. Solution in the transition stage. Under the assumption that the integral in (23) remains convergent as $K$ approaches infinity, the equation requires

$$
\delta=-2 \varepsilon \ln \varepsilon+2 \alpha \varepsilon+b \varepsilon+c \varepsilon^{3 / 2}+O\left(\varepsilon^{2} \ln \varepsilon\right),
$$

where $b$ and $c$ are constants of order unity. The $c$ term is introduced through the $\sqrt{\varepsilon}$ 
term in the integral. That the integral should converge may be reasoned from the observation that in lowest order (21) becomes $F=-\sqrt{1-\tau}$ as $\tau$ approaches unity, implying that an appropriate matching between the initial and transition stages will occur if $f \sim-\sqrt{y}$ as $y$ approaches infinity, a dependence which will cause the square bracket in (23) to vanish (more rapidly than $1 / \sqrt{y^{\prime}}$, it turns out) as $y^{\prime}$ approaches infinity. The transition-stage expansion $f=g+\sqrt{\varepsilon} h+O(\varepsilon \ln \varepsilon)$ also is dictated by (23), where the functions $g(y)$ and $h(y)$ are independent of $\varepsilon$. Substitution of this expansion, along with (24), into (23) yields upon collecting terms of like order in $\varepsilon$ the pair of integral equations

$$
y-g^{2}-2 \ln y-b=\int_{y}^{\infty}\left(\frac{2}{\sqrt{y^{\prime}}}+4 \frac{d g^{\prime}}{d y^{\prime}}\right) \frac{d y^{\prime}}{\sqrt{y^{\prime}-y}}
$$

and

$$
g h+c / 2=\int_{y}^{\infty}\left(1-\frac{d g^{\prime 2}}{d y^{\prime}}-2 \frac{d h^{\prime}}{d y^{\prime}}\right) \frac{d y^{\prime}}{\sqrt{y^{\prime}-y}} .
$$

Equation (25) is a quadratically nonlinear integral equation which is expected to possess a unique solution, properly divergent at $y=0$, for only one value of the constant $b$. For any other value of $b$ the divergence occurs at a value of $y$ different from zero, thereby violating the initial stipulation that ignition occurs at $\tau=1$. There is a kind of translational invariance in the problem, in that matching to the initial stage is achieved for any value of $b$ of order unity. The divergence has been selected to occur at $y=0$ in the stretched variable merely for convenience in calculating the ignition time systematically. In an alternative formulation another value of $b$ may be chosen, and then the value of $\delta$ will change, but the ignition time in the $\tau$ variable, no longer being defined by $y=0$, will remain the same.

After $b$ and $g(y)$ are determined, (26) constitutes a linear integral equation for $h$, the solution to which possesses a singularity at $y=0$, the strength of which may be minimized through proper selection of a value for $c$. Again, matching to the initial stage can be obtained for any value of $c$ of order unity. Minimizing the strength of the singularity effectively extends the validity of the expansion to later times. In the present problem, it may be shown from (29) that $\sqrt{\varepsilon}$ h becomes of the same order as $g$ when $y$ becomes of order $\sqrt{\varepsilon}$, unless the strength of the singularity is minimized, in which case this breakdown of the expansion does not occur until $y$ becomes of order $\varepsilon$. In obtaining values of the ignition time valid through terms of order $\varepsilon^{3 / 2}$ in $\tau$, an expansion that is valid for values of $y$ of order $\sqrt{\varepsilon}$ must be employed, thereby necessitating minimization of the strength of the singularity.

The solutions for $g$ and $h$ provide a solution for $f$, which is expected to increase from $-\sqrt{y}$ to $+\infty$ as $y$ decreases from infinity to zero, thereby exhibiting a smooth transition through the zero-flux time at which $f=0$ and describing a thermal runaway which occurs at $\tau=1$. Upon identifying this thermal runaway with the ignition event as has been done previously [2] the values obtained for $b$ and $c$ serve to determine the ignition time through terms of order $\varepsilon^{3 / 2}$ in $\tau$ by giving the corresponding terms in the asymptotic expansion of $D$.

Expansions of the solutions to (25) and (26) for small and large values of $y$ are useful in seeing characteristics of the results and in completing the numerical calculations of $g$ and $h$. Near $y=0$, the solution to (25) is $g=4 / \sqrt{y}$. In fact, $g=-\sqrt{y}+4 / \sqrt{y}$ would be an exact solution to (25) with $b=8$, except for the presence of the $\ln y$ term. 
For large values of $y$, the expansion

$$
g \sim-\sqrt{y}+(\ln y+b / 2) / \sqrt{y}+O(\ln y / y)
$$

may be developed, while

$$
h \sim c /(2 \sqrt{y})+O(1 / y)
$$

from (26). As $y$ approaches zero, (26) may be employed to show that

$$
h \sim k / y^{3 / 2}+8 /\left[\left(\frac{4}{\pi}-1\right) y\right],
$$

there being a particular value of $c$ that causes the constant $k$ to vanish.

Equations (25) and (26) were solved numerically by a method analogous to that of [2]. Integrations were started at $y=Y$, a large number, making use of (27) and (28) for initial values. In addition, under the assumption that (27) and (28) hold for $y>Y$, the expressions

$$
\begin{aligned}
& \int_{Y}^{\infty}\left(\frac{2}{\sqrt{y^{\prime}}}+4 \frac{d g^{\prime}}{d y^{\prime}}\right) \frac{d y^{\prime}}{\sqrt{y^{\prime}-y}} \\
& =\frac{8}{y}\left[\frac{b}{4}\left(\sqrt{1-\frac{y}{Y}}-1\right)+\ln 2+\frac{1}{2}\left(\sqrt{1-\frac{y}{Y}}-1\right) \ln Y-\ln \left(1+\sqrt{1-\frac{y}{Y}}\right)\right], \\
& \int_{Y}^{\infty}\left(\frac{d h^{\prime}}{d y^{\prime}}\right) \frac{d y^{\prime}}{\sqrt{y^{\prime}-y}}=\frac{c}{2 y}\left(\sqrt{1-\frac{y}{Y}}-1\right)
\end{aligned}
$$

and

$$
\begin{aligned}
\int_{Y}^{\infty}\left(1-\frac{d g^{\prime 2}}{d y}\right) \frac{d y^{\prime}}{\sqrt{y^{\prime}-y}} & \\
= & \frac{4}{\sqrt{y}} \sin ^{-1}\left(\sqrt{\frac{y}{Y}}\right)+(4 \ln Y+2 b-8 \ln 2) \frac{1}{y^{3 / 2}}\left[\sin ^{-1}\left(\sqrt{\frac{y}{Y}}\right)-\sqrt{1-\frac{y}{Y}}\right] \\
& -\frac{8}{y^{3 / 2}}\left\{\sin ^{-1}\left(\sqrt{\frac{y}{Y}}\right)-\sum_{n=o}^{\infty} \frac{(2 n) !(y / Y)^{n+1 / 2}}{\left[2^{n} n !(2 n+1)\right]^{2}}\right\}
\end{aligned}
$$

were derived and employed to evaluate the contributions from the range $y^{\prime}>Y$ to the right-hand sides of (25) and (26). For (25) it is convenient to work with $\hat{g}=g+\sqrt{y}$, the specific finite difference version employed being

$$
\begin{aligned}
\hat{g}_{i}^{2}-2 \sqrt{y_{i}} \hat{g}_{i}+2 \ln y_{i}+b=-4\left\{\frac { 2 } { y _ { i } } \left[\frac{b}{4}\left(\sqrt{1-\frac{y_{i}}{Y}}-1\right)+\right.\right. & \frac{1}{2}\left(\sqrt{1-\frac{y_{i}}{Y}}-1\right) \ln Y \\
\left.-\ln \left(\sqrt{1-\frac{y_{i}}{Y}}+1\right)+\ln 2\right] & \left.+\sum_{j=1}^{i-1} \frac{\hat{g}_{j}-\hat{g}_{j+1}}{\left[\left(y_{j}+y_{j+1}\right) / 2-y_{i}\right]^{1 / 2}}\right\} .
\end{aligned}
$$

An analogous form was used for (26). Adoption of uniform increments in ln y, rather than in $y$, reduced the computation time and improved the accuracy, although even then finer mesh sizes were needed at small values of $y$. It was found that $g$ approaches $4 / \sqrt{y}$ as $y$ approaches zero only if $b=2 \ln (11.98)$ and that $k=0$ in (29) only if $c=26.22$. 
Curves of $g+\sqrt{y}$ and of $h$ are shown in Fig. 1, from which it may be seen that the asymptotic solutions remain reasonably good over relatively large ranges. Also shown are $-g$, the negative of the first approximation to the surface heat flux, and $\theta_{m} / \varepsilon=g^{2}$, the lowest-order approximation to the peak temperature obtained from (11) for $g>0$. It is seen that the peak temperature increases rapidly as thermal runaway is approached.

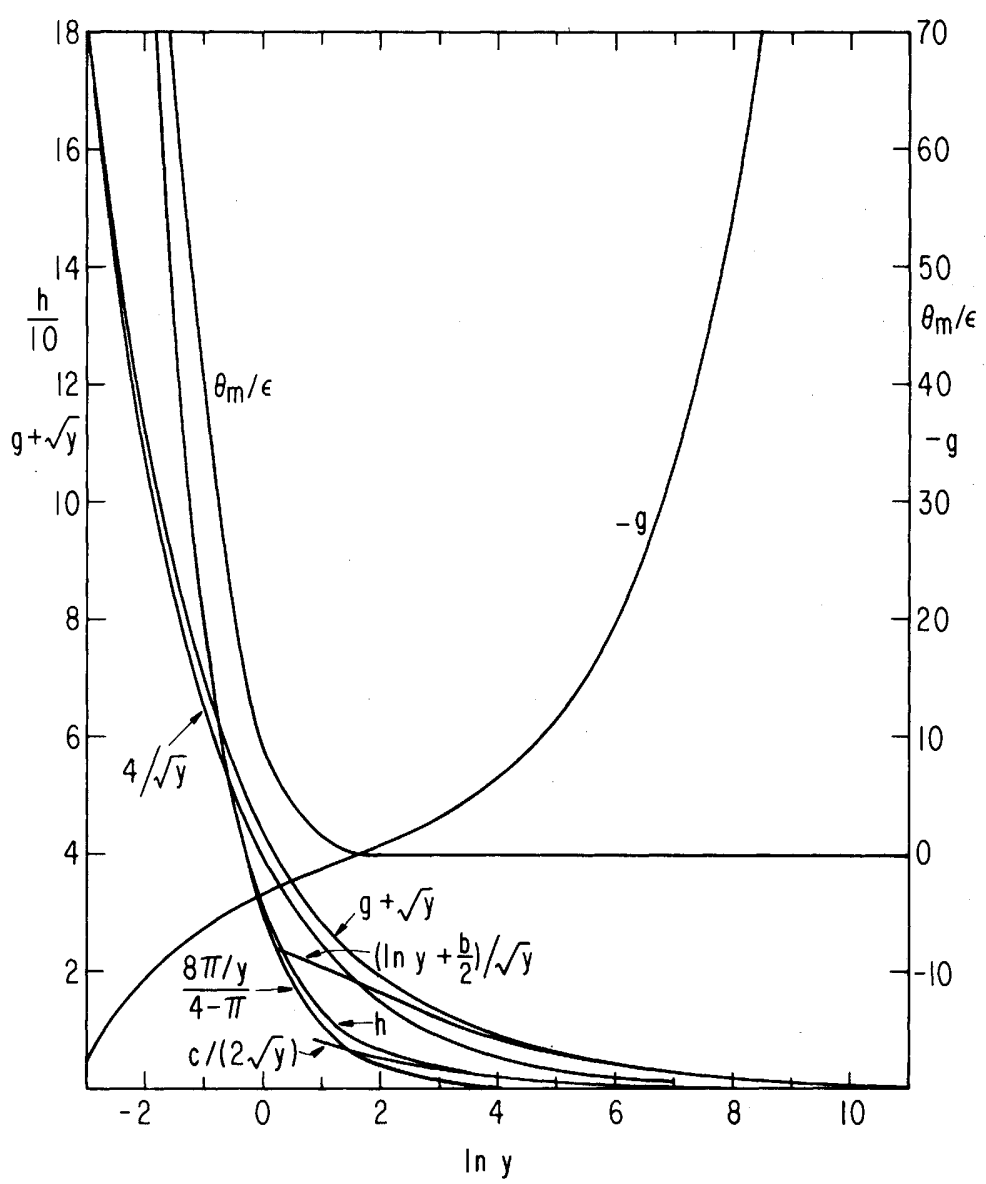

FIG. 1. Transition-stage solution for $g$ and $h$, determining the nondimensional surface heat flux, and for nondimensional peak temperature $\theta_{m} / \varepsilon$.

The time $y_{a}$ at which the heat flux at the surface reaches zero is defined by $f\left(y_{a}\right)=0$. If $a$ denotes the value of $y$ at which $g(y)=0$, then a two term expansion for the time of zero flux is $y_{a}=a-\sqrt{\varepsilon} h(a) / g^{\prime}(a)$, which in the time variable $\tau$ is

$$
\tau_{a}=1-\varepsilon a+\varepsilon^{3 / 2} h(a) / g^{\prime}(a) .
$$

The solution for $g(y)$ shows that $a=5.0$ and that the derivative $g^{\prime}(a)=-0.42$, while the solution for $h(y)$ gives $h(a)=8.1$, resulting in $h(a) / g^{\prime}(a)=-19$, finally giving $\tau_{a}=$ $1-5 \varepsilon-19 \varepsilon^{3 / 2}$. The approximations $a=b=\sqrt{c}=-h(a) / 4 g^{\prime}(a)=2 \ln (12)=5.0$ may lie within the accuracy of the calculations, although $b=4.97$ and other values quoted above seem more reliable.

Since the problem is of the evolution type, in the sense that events occurring later than a given time $\tau$ do not influence the solution at time $\tau$ or earlier, an alternative 
approach to the calculation of the time of zero heat flux is to adjust $D$ so that $f=0$ at $y=0$. Specifically, with the expansion given in (24), the constant $b$ is selected in (25) to obtain $g=0$ at $y=0$, and the constant $c$ is selected in (26) to obtain $h=0$ at $y=0$. In this approach $\tau_{a}=1$ by the modified definition. Numerical solutions were obtained with these conditions imposed, and it was found that when the results were expressed in terms of the previous variables, the solution was the same, within the accuracy of the numerical calculation, until the time of zero flux. Therefore the result obtained from (34) appears to be insensitive to the fact that it is based on a solution that extends beyond the time of zero flux.

Other characteristics of the solution also may be computed. For example, temperature profiles in the reaction zone are obtained from (14) by putting $F=\sqrt{\varepsilon} f$. Temperature profiles in the inert zone can be computed in principle from a generalization [4] of the Abel inversion of (7), by evaluating an integral numerically, with (18) employed for $\varphi_{\zeta}$. Thus, all desired properties of the transition stage solution become accessible. The qualitative similarity to the problem [2] of constant flux encompasses the two-stage, two-zone aspect as well as the occurrence of runaway in a transition stage for a stretched dependent variable. The main physical difference, producing greater complexity, is the occurrence in the present problem of reaction in the inner zone, beginning at time zero instead of only at the end of an inert stage. This strengthens the interaction between the initial and transition stages through the accumulating effect of apparent surface temperature due to the chemical reaction in the first stage.

6. Results and comparisons. The results having greatest interest are the times of zero heat-flux and of thermal runaway. It has been conventional [1] to employ as the nondimensional time variable $\hat{\tau}=D \tau /\left(2 \pi \varepsilon^{2}\right)$ and to use $\theta_{i}=1 / \varepsilon$ as a nondimensional activation energy. In the variable $\hat{\tau}$, the blowup time of runaway is $\hat{\tau}_{b}=D /\left(2 \pi \varepsilon^{2}\right)$, and the zero-flux time is $\hat{\tau}_{a}=D \tau_{a} /\left(2 \pi \varepsilon^{2}\right)$. Use of (24) and (34), along with $D=1+\delta$, in these definitions yields

$$
\hat{\tau}_{a}=\frac{1}{2 \pi \varepsilon^{2}}\left\{1-2 \varepsilon \ln \varepsilon+(b-a+2 \alpha) \varepsilon+\left[c+h(a) / g^{\prime}(a)\right] \varepsilon^{3 / 2}+O\left(\varepsilon^{2} \ln \varepsilon\right)\right\}
$$

and

$$
\hat{\tau}_{b}=\frac{1}{2 \pi \varepsilon^{2}}\left[1-2 \varepsilon \ln \varepsilon+(b+2 \alpha) \varepsilon+c \varepsilon^{3 / 2}+O\left(\varepsilon^{2} \ln \varepsilon\right)\right]
$$

Within the accuracy of the calculations performed herein, these results may be written as

$$
\hat{\tau}_{a}=\frac{1}{2 \pi \varepsilon^{2}}\left[1-2 \varepsilon \ln \varepsilon+2 \alpha \varepsilon+7 \varepsilon^{3 / 2}+O\left(\varepsilon^{2} \ln \varepsilon\right)\right]
$$

and

$$
\hat{\tau}_{b}=\frac{1}{2 \pi \varepsilon^{2}}\left[1-2 \varepsilon \ln \varepsilon+(4.97+2 \alpha) \varepsilon+26.22 \varepsilon^{3 / 2}+O\left(\varepsilon^{2} \ln \varepsilon\right)\right] .
$$

It is of interest to compare these formulas with those of Averson, Barzykin and Merzhanov [5], obtained by correlating results of numerical integrations of (3) and (4), viz.

$$
\hat{\tau}_{a}=\left(0.20 / \varepsilon^{2}\right)(1+5 \varepsilon)(1+\alpha \varepsilon)
$$


and

$$
\hat{\tau}_{b}=\left(0.20 / \varepsilon^{2}\right)(1+8 \varepsilon)(1+\alpha \varepsilon),
$$

stated to be accurate within $7 \%$ for $\varepsilon$ between $\frac{1}{3}$ and $\frac{1}{30}$ and $\alpha$ between 0 and 0.9 .

The Zeldovich approximation for the time required for the surface heat flux to reach zero and for ignition to occur is [1] $2 \pi \varepsilon^{2} \hat{\tau}_{a}=2 \pi \varepsilon^{2} \hat{\tau}_{b}=1$. Table 1 lists zero-flux and blowup times, referred to their Zeldovich values, as obtained from (37) to (40) and also from another numerical integration $[6]^{1}$ of the partial differential equations, performed independently but carried only up to the zero-flux time. The four-term expansions are those given in (37) and (38); the three term expansions were obtained by omitting the $\varepsilon^{3 / 2}$ term in (37) and (38) and are included to provide an indication of the accuracy of the asymptotic results. That the percentage differences between the three term and four term expansions are appreciable for $\theta_{i}=5$ and 10 suggests that the asymptotic expansion may lack accuracy at these values of $\theta_{i}$. According to the rough principle that any term in an asymptotic expansion should be discarded if its magnitude exceeds that of the preceding term, only a three-term expansion for $\hat{\tau}_{b}$ is justified for $\varepsilon>0.04$, and only a two term expansion for $\varepsilon>0.08$. In general, the asymptotic expansions are of well justified accuracy only for $\varepsilon \leqslant 0.05$. In this respect, the results differ from earlier ones [2] in which the expansions held to much larger values of $\varepsilon$. It seems likely that this difference is related to the stronger interaction between the initial and transition stages in the present problem, manifest in the appearance of logarithmic terms. The four-term expansion may be accurate to $\theta_{i}=5$ because asymptotic expansions often are good beyond their ranges of justification, but this cannot be ascertained solely by studying asymptotic expansions.

Guidance concerning the accuracy to be expected from the asymptotic expansion may be sought by comparing the asymptotic results with those obtained from numerical

TABLE 1

Comparisons of Zero flux and blowup times.

\begin{tabular}{|c|c|c|c|c|c|c|c|}
\hline \multirow{3}{*}{$\begin{array}{c}\text { Nondimentional } \\
\text { Activation Eneray } \\
\theta_{i}=1 / 8\end{array}$} & \multicolumn{7}{|c|}{$\begin{array}{l}\text { Zero-Flux Time Referred to Zeldovich Approximation } \\
\qquad 2 \pi \varepsilon \hat{r}_{a}=D \tau_{a}\end{array}$} \\
\hline & \multicolumn{4}{|c|}{$\alpha=0$} & \multicolumn{3}{|c|}{$\alpha=1$} \\
\hline & $\begin{array}{l}\text { Four-term } \\
\text { expansion }\end{array}$ & $\begin{array}{l}\text { Three-term } \\
\text { expansion }\end{array}$ & Ref. [5] & Ref. [6] & $\begin{array}{l}\text { Four-term } \\
\text { expansion }\end{array}$ & $\begin{array}{l}\text { Three-term } \\
\text { expansion }\end{array}$ & Ref. [5] \\
\hline $\begin{array}{r}5 \\
10 \\
25 \\
100\end{array}$ & $\begin{array}{l}2.27 \\
1.68 \\
1.32 \\
1.099\end{array}$ & $\begin{array}{l}1.64 . \\
1.46 \\
1.26 \\
1.092\end{array}$ & $\begin{array}{l}2.51 \\
1.88 \\
1.51 \\
1.32\end{array}$ & $\begin{array}{l}1.61 \\
1.29 \\
1.096\end{array}$ & $\begin{array}{l}2.67 \\
1.88 \\
1.40 \\
1.12\end{array}$ & $\begin{array}{l}2.04 \\
1.66 \\
1.34 \\
1.11\end{array}$ & $\begin{array}{l}3.01 \\
2.07 \\
1.57 \\
1.33\end{array}$ \\
\hline \multirow{3}{*}{100} & \multicolumn{7}{|c|}{$\begin{array}{l}\text { Blowup Time Referred to Zeldovich Approximation } \\
\qquad 2 \pi z t_{b}=D t_{b}\end{array}$} \\
\hline & \multicolumn{4}{|c|}{$\alpha=0$} & \multicolumn{3}{|c|}{$\alpha=1$} \\
\hline & $\begin{array}{l}\text { Four-term } \\
\text { expansion }\end{array}$ & $\begin{array}{l}\text { Three-term } \\
\text { expansion }\end{array}$ & Ret: [5] & & $\begin{array}{l}\text { Four-term } \\
\text { expansion }\end{array}$ & $\begin{array}{l}\text { Three-term } \\
\text { expansion }\end{array}$ & Ref. [5] \\
\hline 5 & 4.98 & 2.64 & 3.27 & & 5.38 & 3.04 & 3.92 \\
\hline 10 & 2.79 & 1.96 & 2.26 & & 2.98 & 2.16 & 2.49 \\
\hline 25 & 1.67 & 1.46 & 1.66 & & 1.75 & 1.54 & 1.73 \\
\hline 100 & 1.16 & 1.14 & 1.36 & & 1.18 & 1.16 & 1.37 \\
\hline
\end{tabular}

\footnotetext{
${ }^{1}$ See also the work of O. B. Sidonskii quoted in [6b].
} 

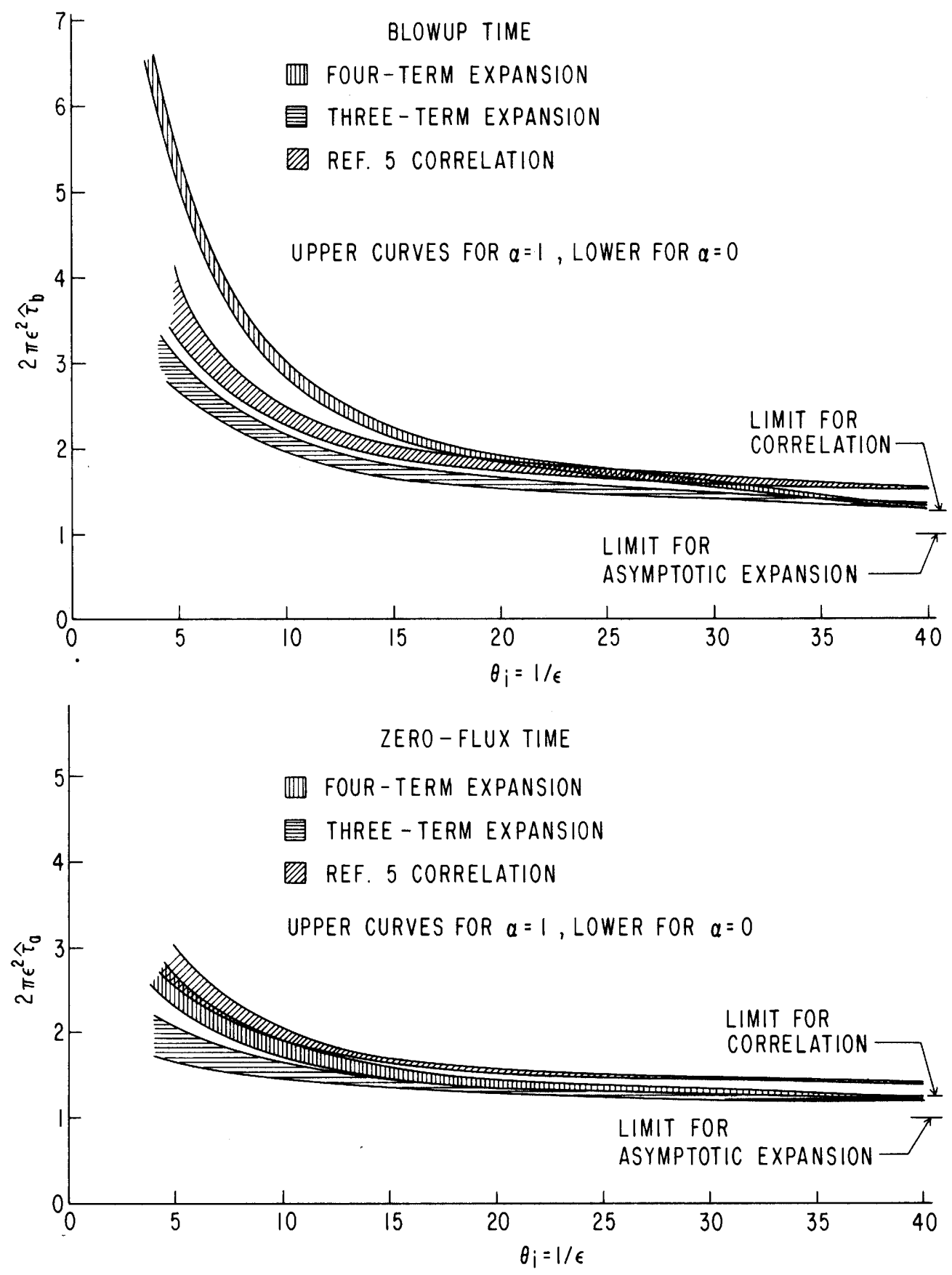

FIG. 2. Comparison of (a) ignition times and of (b) times of surface adiabaticity, obtained from the asymptotic analysis and from earlier results based on numerical integrations.

integration of the partial differential equations. Unfortunately, comparison of results of [5] and [6] reveals uncertainties in the numerical integrations that are comparable with the difference between the three-term and four-term expansions. For example, entries in Table 1 for the zero-flux time at $\theta_{i}=10$ and at $\theta_{i}=25$, where (39) and (40) are claimed to be good, reveal differences between the correlation and the numerical 
integration of [6] that are on the order of $15 \%$, considerably greater than the stated error of the correlations [5]. Results of the four term expansion for $\hat{\tau}_{a}$ with $\alpha=0$ lie between those of the two numerical studies but quite close to the results of [6]. This suggests that the four-term expansion may represent the best results currently available, at least for $\varepsilon \geq 0.1$.

The comparison of (37) to (40) is shown graphically in Fig. 2. It is seen that for $\theta_{i} \leqslant 20$ the correlation for $\hat{\tau}_{b}$ lies between the three-term and four-term expansion, while the correlation for $\hat{\tau}_{a}$ lies above the four-term expansion. It is uncertain whether the correlation or the four-term expansion is better in this range. At larger values of $\theta_{i}$ the asymptotic formulas certainly are more accurate than the correlation formulas. Since numerical integrations of the partial differential equations become progressively more tedious as $\varepsilon$ decreases, the asymptotic results are especially useful at small $\varepsilon$.

In one respect, the asymptotic analysis appears to provide results that are useful over the entire range of $\varepsilon$ values of practical interest. This concerns the dependences of critical times on the parameter $\alpha=\left(T_{s}-T_{i}\right) / T_{s}$, which describes the influence of the difference between Arrhenius and Frank-Kamenetskii kinetics. The dependences on $\alpha$ in (37) and (38) are small, of order $\varepsilon$, but not entirely negligible, since differences between critical times for extreme values of $\alpha$ amount to five or ten percent for representative values of $\varepsilon$. In the asymptotic expansions, the neglected terms of order $\varepsilon^{2} \ln \varepsilon$ are independent of $\alpha$, the first $\alpha$-dependent correction beyond $2 \alpha \varepsilon$ being of order $\varepsilon^{2}$. Thus, the relatively strong tendency toward divergence of the expansion does not occur for the $\alpha$ terms, and the influence of $\alpha$ on $\hat{\tau}$ is expected to be given accurately by (37) and (38) over a wide range of $\varepsilon$. This suggests that (39) and (40) tend to underestimate the effect of $\alpha$ somewhat for small $\varepsilon$ and that improved formulas in the range quoted $\left(\frac{1}{3}<\varepsilon<\frac{1}{30}\right)$ may be

$$
\hat{\tau}_{a}=\left(0.20 / \varepsilon^{2}\right)(1+5 \varepsilon+5 \alpha \varepsilon / \pi)
$$

and

$$
\hat{\tau}_{b}=\left(0.20 / \varepsilon^{2}\right)(1+8 \varepsilon+5 \alpha \varepsilon / \pi) .
$$

This stronger dependence also seems to agree better with a numerical result quoted in [6].

7. Concluding remarks. It is of interest to enquire whether improved accuracy can be obtained through further asymptotic developments. The nonuniformity in time in the present approach is evident in the thermal runaway that occurs at $y=0$. Of course, infinite temperatures and fluxes are not predicted by the original equations, and the runaway signifies failure of the transition-stage expansion. That this failure occurs when $y$ becomes of order $\varepsilon$ is clear from the fact that the small-y behavior $g=4 / \sqrt{y}$ then produces an $f$ of order $1 / \sqrt{\varepsilon}$ and therefore an $F$ of order unity, not small. Typically the breakdown begins relatively soon in the transition stage, as may be seen by comparing the asymptotic forms of $g$ and $h$ for small $y$; the higher-order term $\sqrt{\varepsilon} h$ becomes larger than $g$ if $y<53.6 \varepsilon$, which even for $\varepsilon=0.04$ excludes the range $y<2$. It is possible to identify an ignition stage in which $y / \varepsilon$ is of order unity, and although analysis of (20) in this stage would be straightforward, the solution would not be meaningful since it follows from (3) that the stretching would cause time derivatives to appear explicitly in lowest order in the equations describing the reaction zone.

Perhaps a proper description of the ignition stage can be obtained through generalizations of the promising methods of Hermance [7] and Kassoy [8] but this is unclear because as yet those methods have not been developed for problems involving 
partial differential equations. For procedures extending into the ignition stage or beyond, an ignition criterion different from thermal runaway will have to be developed if comparisons with previous results, such as (39) and (40), are to be made. At present it is unclear whether significantly improved predictions of ignition times can be obtained from asymptotic analysis for the problem under consideration. Possibly the approach adopted herein is the optimum.

From the mathematical viewpoint, the study of asymptotically valid integral equations appears to be a relatively unexplored field, worthy of further research. Casting asymptotic problems in the form of integral equations sometimes can simplify them significantly, although obtaining asymptotic solutions to the integral equations may present unique challenges. In this respect, it may be noted that the present problem is of a different type than the integral-equation problems studied previously [9], [10], in that an asymptotic expansion with respect to a parameter is sought, rather the asymptotic behavior for large time.

From the practical viewpoint, it may be noted that the analysis is applicable directly to ignition of a reactive solid suddenly brought into contact with a hot plate, if the thermal responsivity $(\lambda \rho c)$ of the plate is large compared with that of the solid. There are also problems in ignition of gases for which the analysis may be used. For example, ignition of an initially quiescent, premixed gaseous combustible exposed to a step in surface temperature is described by exactly these same equations if $\rho \lambda$ is constant and a Howarth transformation is introduced. The isothermal boundary of elevated temperature which serves as the ignition source (for example, a heated metal having high thermal responsivity) may be nonplanar, provided that its radius of curvature is large compared with the thickness of the thin heated layer that develops in the fuel prior to ignition. Also, ignition of a combustible gas in steady flow over a heated, isothermal, flat plate presents essentially the same analytical problem. It should be possible to employ directly many of the results developed herein for such related problems. The general methodology is capable of being extended to somewhat more complex problems, as shown in the appendices, from which it may be seen that depending on specifics of the problem, the task that emerges is to solve numerically integral equations, ordinary differential equations or partial differential equations.

Appendix A. Effect of reactant consumption. To account for effects of reactant consumption with a first-order reaction, (3) and (4) are replaced by the system

$$
\begin{gathered}
\frac{\partial \theta}{\partial \tau}-\frac{\partial^{2} \theta}{\partial \xi^{2}}=\frac{D}{2 \pi \varepsilon^{2}}(1-Y) e^{\theta /(1+\alpha \varepsilon \theta)}=\frac{1}{\gamma}\left(\frac{\partial Y}{\partial \tau}-\frac{1}{L} \frac{\partial^{2} Y}{\partial \xi^{2}}\right), \\
\theta(\xi, 0)=\theta(\infty, \tau)=-\varepsilon^{-1}, \quad \theta(0, \tau)=Y(\xi, 0)=Y(\infty, \tau)=0=(\partial Y / \partial \xi)_{\xi=0},
\end{gathered}
$$

where $1-Y$ is the reactant concentration divided by its initial value. The two additional parameters that appear are $\gamma=\rho c R T_{s}^{2} /(E q)$, a measure of the magnitude of the effect of reactant consumption, and the Lewis number $L$, the ratio of heat to reactant diffusivities. This problem was studied previously for nondiffusive reactants $(L=\infty)$ by numerical integration of the partial differential equations [5]. For $L=\infty$, the last boundary condition in (A2) is deleted; for finite $L$ this condition represents vanishing of the diffusive flux of reactant at the surface. The asymptotic solutions to (A1) and (A2) will be studied first for nondiffusive reactants in the distinguished limit in which $\alpha$ and $\Gamma=\gamma / \varepsilon^{2}$ are of the order unity, and second for $L$ of order unity (representative of gases) in the distinguished limit having $\gamma / \varepsilon$ of order unity. 
Condensed reactants, $L=\infty$. In the first case there are two regions, an outer region of unsteady transport where, in a first approximation, $Y=0$ and $\theta=\theta_{l}$, defined above (5), and an inner reaction zone, where $Y$ and the temperature increment $\psi$, which appears in (9), are given in a first approximation by the equations

$$
-\frac{\partial^{2} \psi}{\partial \eta^{2}}=\frac{1}{2}(1-Y) e^{\psi-\eta / \sqrt{\tau}}=\frac{\pi}{\Gamma} \frac{\partial Y}{\partial \tau},
$$

subject to the conditions $Y(\eta, 0)=\psi(0, \tau)=(\partial \psi / \partial \eta)_{\eta=\infty}=0$. The condition for large $\eta$ is a first approximation to the matching condition, and $D$ has been set equal to unity because the requirement that ignition occurs at $\tau=1$ has been omitted in this simplified approach that is applicable in lowest order.

The ignition time is the largest value of $\tau$ for which the solution to (A3) with its specified conditions exists. In general, (A3) is a one parameter system of partial differential equations that should be integrated numerically, starting at $\tau=0$ and marching forward in $\tau$ until reaching an ignition time, $\tau_{\mathrm{ig}}(\Gamma)$, at which $\partial \psi / \partial \tau$ goes to infinity at some value of $\eta$. It is clear from (A3) that as the parameter measuring the strength of the reactant consumption, $\Gamma$, increases, the ignition time, $\tau_{\text {ig }}$, increases, and a point eventually is reached at which $\tau_{\text {ig }}$ goes to infinity. Therefore $\tau_{\mathrm{ig}}\left(\Gamma_{c}\right)=\infty$ defines a critical value, $\Gamma_{c}$, of $\Gamma$ above which a smooth solution without a well-defined ignition event will be found for all $\tau$. Notice from (A3) that at $\eta=0$ the value of $Y$ is given by $Y_{s}(\tau)=1-\exp (-\tau \Gamma /(2 \pi))$ for all $\Gamma$.

For small values of $\tau$, the solution to (A3) is

$$
\psi=(\tau / 2)\left(1-e^{-\mu}\right), \quad Y=(\tau \Gamma / \pi) \mu^{2} \Gamma(-2, \mu),
$$

where $\mu=\eta / \sqrt{\tau}$ and the incomplete gamma function is $\Gamma(-2, \mu)=\int_{\mu}^{\infty} z^{-3} e^{-z} d z$. As $\mu$ approaches zero, $\mu^{2} \Gamma(-2, \mu)$ approaches $\frac{1}{2}$. This small- $\tau$ solution may be useful for starting the numerical integration, which might best be performed in the variables $\mu$ and $\tau$, in which the equation is

$$
-\frac{\partial^{2} \psi}{\partial \mu^{2}}=\frac{\tau}{2}(1-Y) e^{\psi-\mu}=\frac{\pi}{\Gamma}\left(\tau \frac{\partial Y}{\partial \tau}-\frac{\mu}{2} \frac{\partial Y}{\partial \mu}\right)
$$

with conditions $Y(\mu, 0)=\psi(0, \tau)=(\partial \psi / \partial \mu)_{\mu=\infty}=0$.

Notice that for small values of $\Gamma, Y$ is proportional to $\Gamma$ and the effect of reactant consumption is negligible in (A3). If $\Gamma=0$ then $Y=0$ and (A3) may be integrated to show that

$$
(\partial \psi / \partial \eta)_{\eta=0}=1 / \sqrt{\tau}-\sqrt{(1-\tau) / \tau},
$$

which becomes imaginary for $\tau>1$. Therefore $\tau=1$ is the first approximation to the ignition time for $\Gamma=0$. This is in agreement with the Zeldovich result discussed in the main text.

For intermediate values of $\Gamma$, numerical integration is necessary. For large values of $\Gamma$, the variables $\psi_{1}=\psi \Gamma, \eta_{1}=\eta \sqrt{\Gamma}$ and $\tau_{1}=\tau \Gamma$ are useful, since in these variables when $\Gamma$ is infinite (3) becomes

$$
-\frac{\partial^{2} \psi_{1}}{\partial \eta_{1}^{2}}=\frac{1}{2}(1-Y) e^{-\eta_{1} / \sqrt{\tau_{1}}}=\pi \frac{\partial Y}{\partial \tau_{1}},
$$

with the boundary conditions $Y\left(\eta_{1}, 0\right)=\psi_{1}\left(0, \tau_{1}\right)=\left(\partial \psi_{1} / \partial \eta_{1}\right) \eta_{1}=\infty=0$. The thermal runaway, produced by $e^{\psi}$, no longer is present, and the solution will exist for all time. The second of these last equations may be integrated first to yield

$$
Y=1-\exp \left[-\left(\eta_{1}^{2} / \pi^{2}\right) \Gamma(-2, \mu)\right]
$$


which may be substituted into the first equation, giving a two-point boundary-value problem for a second-order ordinary differential equation for $\psi_{1}$.

Previously reported [5] correction factors for reactant consumption in (39) and (40) are $(1+\varepsilon \Gamma)$ and $\left(1+8 \varepsilon^{2} \Gamma^{3 / 2}\right)$, respectively. The present considerations suggest that the effect may be larger than these factors would indicate. Reactant consumption is a small correction only when $\gamma$ is small compared with $\varepsilon^{2}$.

Gaseous Reactants, $\boldsymbol{L}=\boldsymbol{O}(\mathbf{1})$. In the second case, there is an inner reaction layer where $1-Y$ is of order unity and spatial changes in $Y$ are of order $\varepsilon$. The reactant diffuses toward this layer from a thicker layer of unsteady transport, in which, again, $\theta=\theta_{1}$, while now

$$
\frac{\partial Y}{\partial \tau}-\frac{1}{L} \frac{\partial^{2} Y}{\partial \xi^{2}}=0
$$

From (A9), the apparent surface concentration, $Y_{s}(\tau)=Y(0, \tau)$, is related to the diffusive flux by the equation [4]

$$
Y_{s}(\tau)=-\left.\frac{1}{\sqrt{\pi L}} \int_{0}^{\tau} \frac{\partial Y\left(\xi, \tau^{\prime}\right)}{\partial \xi}\right|_{\xi=0} \frac{d \tau^{\prime}}{\sqrt{\tau-\tau^{\prime}}}
$$

In the thin reaction zone $Y$ differs from $Y_{s}(\tau)$ by a quantity of order $\varepsilon$. The temperature increment $\psi$ above the inert value and the variation of $Y$ across the reacting layer are given by the equations

$$
-\frac{\partial^{2} \psi}{\partial \eta^{2}}=\frac{1}{2}\left(1-Y_{s}\right) e^{\psi-\eta / \sqrt{\tau}}=-\frac{1}{\gamma L} \frac{\partial^{2} Y}{\partial \eta^{2}},
$$

in the first approximation. Equation (A11) along with the last boundary condition in (A2) gives

$$
\frac{\partial \psi}{\partial \eta}-\frac{1}{\gamma L} \frac{\partial Y}{\partial \eta}=\left(\frac{\partial \psi}{\partial \eta}\right)_{\eta=0}
$$

Since matching requires $(\partial \psi / \partial \eta)_{\eta=\infty}=0$ in the first approximation, (A12) shows through matching that in (A10)

$$
\frac{1}{\sqrt{L}}\left(\frac{\partial Y}{\partial \xi}\right)_{\xi=0}=\frac{1}{\sqrt{L}} \frac{1}{\varepsilon \sqrt{\pi}}\left(\frac{\partial Y}{\partial \eta}\right)_{\eta=\infty}=-\frac{\gamma}{\varepsilon} \sqrt{\frac{L}{\pi}}\left(\frac{\partial \psi}{\partial \eta}\right)_{\eta=0} .
$$

A first integral of the first equality in (A11) produces

$$
\left(\frac{\partial \psi}{\partial \eta}\right)_{\eta=0}=\frac{1}{\sqrt{\tau}}-\sqrt{Y_{s}+(1-\tau) / \tau}
$$

Substitution of (A13) and (A14) into (A10) then yields the integral equation

$$
Y_{s}(\tau)=\frac{C}{\sqrt{\pi}} \int_{0}^{\tau}\left[\frac{1}{\sqrt{\tau^{\prime}}}-\sqrt{Y_{s}\left(\tau^{\prime}\right)+\left(1-\tau^{\prime}\right) / \tau^{\prime}}\right] \frac{d \tau^{\prime}}{\sqrt{\tau-\tau^{\prime}}}
$$

involving the parameter $C=(\gamma / \varepsilon) \sqrt{L / \pi}$ which measures the effect of reactant consumption.

If $C$ is small then introduction of the expansion $Y_{s}=Y_{1} C+Y_{2} C^{2}+\cdots$ produces an integral formula for $Y_{1}(\tau)$. For small $C$, equation (A14) then shows that the surface heat flux becomes imaginary at $\tau=1$ in the lowest approximation. This gives $\tau=1$ as the first approximation for the ignition time. Use of the expansion in $C$ in (A14) then 
produces $\tau_{\mathrm{ig}}=1+C Y_{1}(1)$ as a second approximation for the ignition time for small $C$. It may be shown that $Y_{1}(1)=(\pi-2) / \sqrt{\pi}$.

For intermediate values of $C$ (A15) must be integrated numerically. Results of the numerical integration give the curve of ignition time shown in Fig. 3. The ignition time goes to infinity at a critical value of $C$, approximately equal to 0.89 . For values of $C$

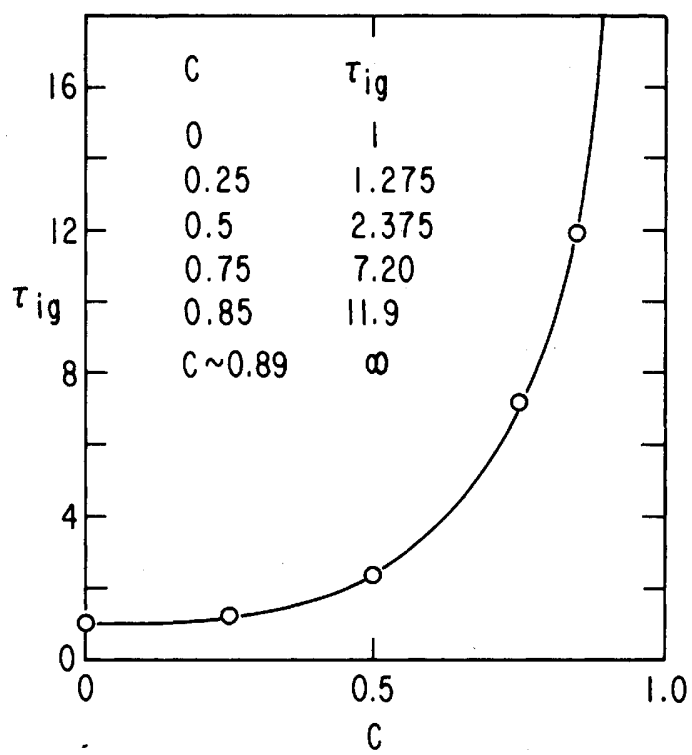

FIG. 3. Dependence of nondimensional ignition time on reactant consumption parameter for ignition of a gaseous combustible having a Lewis number of order unity.

larger than this critical value (A15) should be written in terms of $\tau_{1}=C \tau$ as a new variable, and the expansion $Y_{s}=y_{0}+y_{1} C^{-1}+\cdots$ may be introduced giving

$$
y_{0}\left(\tau_{1}\right)=\frac{1}{2 \sqrt{\pi}} \int_{0}^{\tau_{1}} \frac{\left[1-y_{0}\left(\tau_{1}^{\prime}\right)\right] \sqrt{\tau_{1}^{\prime}}}{\sqrt{\tau_{1}-\tau_{1}^{\prime}}} d \tau_{1}^{\prime}
$$

as a linear integral equation to be solved for the first approximation to the time history of reactant concentration in the reaction zone for large values of $C$. The solution to (A16) of course is smooth and exists for all time.

For $L=\infty$, significant influence of reactant consumption in producing changes in the qualitative character of the solution occurred at relatively small values of $\gamma$, of order $\varepsilon^{2}$. For $L=O(1)$, the effect of reactant consumption is less severe in that the corresponding qualitative changes occur at larger values of $\gamma$, of order $\varepsilon$. The reason for the difference is that diffusion of the reactant into the reaction zone lessens the influence of reactant depletion by replenishing consumed reactant.

Appendix B. Ignition by a hot catalytic surface. Title problem, considered previously [11], is defined by (A1) and (A2) with the last boundary condition replaced by $Y(0, \tau)=1$. As in Appendix A, there will be an outer region of unsteady transport and an inner reaction zone. We consider the distinguished limit in which $\gamma$ is ordered such that significant reactant consumption due to the chemical reaction occurs in the reaction layer. Without reactant consumption, the concentration distribution would be $1-Y=$ $\operatorname{erf}(\xi \sqrt{L /(4 \tau)})$, which is approximately $\xi \sqrt{L /(\pi \tau)}$ in the reaction region. The ordering 
of $\gamma$ will be such that reaction decreases the concentration by an amount of this same order.

In the reaction region, the time derivatives are of higher order, and the stretching leads to $\partial^{2} \theta / \partial \xi^{2}=\left(\partial^{2} Y / \partial \xi^{2}\right) /(\gamma L)$, the second integral of which gives, as a first approximation,

$$
Y=1+\gamma L[\theta+(\xi / \sqrt{\pi \tau})(1 / \varepsilon-1 / \gamma \sqrt{L})] .
$$

Boundary conditions at $\xi=0$ and matching to the outer solution have been used here. The equation for $\psi$ in the inner region that then results from (A1) by use of (B1) is

$$
\partial^{2} \psi / \partial z^{2}=-\sigma(z-B \psi) e^{\psi-z},
$$

where $z=\xi /(\varepsilon \sqrt{\pi \tau}), \sigma=D \tau \sqrt{L} /(2 \varepsilon)$ and $B=\gamma \varepsilon \sqrt{L}$. The boundary and matching conditions in the first approximation require $\psi=0$ at $z=0$ and $\partial \psi / \partial z=0$ at $z=\infty$. Note that now the scaling results in reactant consumption being significant only for very large values of $\gamma$, namely $\gamma$ of order $1 / \varepsilon$ ( $B$ of order unity).

Equation (B2) with its boundary conditions will possess a solution only for $\sigma$ below a critical value that depends on $B, \sigma_{\mathrm{ig}}(B)$, which defines the ignition time. In general, numerical integration of (B2) would be needed to generate the curve of $\sigma_{\mathrm{ig}}(B)$. In the special case $B=1$ a first integral results in $(\partial \psi / \partial z)_{z=0}=1-\sqrt{1-2 \sigma}$, which becomes imaginary at $\sigma=\frac{1}{2}$, thereby giving $\sigma_{\mathrm{ig}}(1)=\frac{1}{2}$. Of greater interest is $\sigma_{\mathrm{ig}}(0)$, the ignition time for a negligible effect of reactant consumption. Since $\sigma_{\mathrm{ig}}(B)$ is an increasing function, we know that $\sigma_{\text {ig }}(0)$ is less than $\frac{1}{2}$, but still of order unity; a numerical integration has given $\sigma_{\mathrm{ig}}(0) 0.239$. Letting $D=1$, one may use the rough approximation $\tau_{\mathrm{ig}}=\varepsilon / \sqrt{L}$ for the ignition time in this problem.

Acknowledgment. We wish to thank M. Kindelan, T. Niioka and T. Mitani for obtaining the numerical solutions to (25), (26) and (A15).

\section{REFERENCES}

[1] A. G. Merzhanov And A. E. Averson, The Present State of the Thermal Ignition Theory: An Invited Review, Combustion and Flame, 16 (1971), pp. 89-124.

[2] A. LIÑÁN AND F. A. Williams, Theory of ignition of a reactive solid by constant energy flux, Combustion Sci. Tech., 3 (1971), pp. 91-98.

[3] - Radiant ignition of a reactive solid with in-depth obsorption, Combustion and Flame, 18 (1972), pp. 85-97.

[4] H. S. Carslaw ANd J. C. JAeger, Conduction of Heat in Solids, 2nd Ed., Oxford University Press, Oxford, 1959.

[5] A. E. AVERSON, V. V. BARzyKin AND A. G. MERzhanov, Laws of ignition of condensed explosive systems with perfect heat transfer at the surface and allowance for burnup, Inž. Fiz. Ž., 39, no. 2 (1965), pp. 245-249.

[6a] V. N. VILYUNOV AND O. B. SIDONSKII, Theory of ignition of condensed systems by an incandescent surface, Dokl. Akad. Nauk SSSR, 152 (1963), pp. 131-133.

[6̄i] R. S. BOORKINA AND V. N. VILYUNOV, Asymptotic analysis of a problem of ignition of reactive material with surface heating, Ž. Prikl. Meh. Teh. Fiz. 100, no. 6 (1976), pp. 96-102.

[7] C. E. HERMANCE, Ignition analysis of adiabatic homogeneous systems including reactant consumption, AIAA J., 11 (1973), pp. 1728-1731.

[8] D. R. KASSOY, A theory of adiabatic homogeneous explosion from initiation to completion, Combustion Sci. Tech., 10 (1974), pp. 27-35.

[9] J. M. GREENBERG AND F. HOPPENSTEADT, Asymptotic behavior of solutions to a population equation, this Journal, 28 (1975), pp. 662-674.

[10] W. E. Olmstead AND R. A. HANDElsman, Asymptotic solution to a class of nonlinear volterra integral equations II, this Journal, 30 (1976), pp. 180-189.

[11] A. M. Grishin, The ignition of a hot catalytic surface, Inž. Fiz. Ž., 13, no. 4 (1967), pp. 514-521. 